\title{
Morphological and Kinetic Embryological Criteria and Correlation with Aneuploidy Rates: How Might they Be Used to Choose the Best IVF Embryo for Transfer?
}

Coates $A^{1,2}$, Coate $B^{1}$, Holmes $L^{1}$ and Griffin DK ${ }^{2 *}$

${ }^{1}$ Oregon Reproductive Medicine, 2222 NW Lovejoy, Suite 304, Portland, Oregon, 97210, USA

${ }^{2}$ School of Biosciences, University of Kent, Canterbury, Kent, CT2 7NJ, UK

\begin{abstract}
Objective: To test the hypothesis that embryos reaching a certain developmental stage at a fixed time point (indicative of growth rate) and those of better morphology are more likely to be euploid than those reaching an earlier stage at the same point and those with poorer morphology. To test the hypothesis that quality of the inner cell mass (ICM) and trophectoderm (TE) independently predict ploidy status. 5 specific age groups were tested

Design: Observational research study, retrospective analysis

Setting: Clinical IVF laboratory, comprehensive chromosome screening (CCS) outsourced to specialist laboratory

Patients: Those undergoing IVF with Comprehensive Chromosome Testing (CCS) by array Comparative Genomic Hybridization (aCGH).

Interventions: All embryos grown to day 5 and 6 . Those that formed advanced blastocysts were biopsied and cells were analyzed using aCGH

Main Outcome Measures: developmental stage reached by a fixed time point and morphology of whole embryos and quality of ICM and TE; correlation to rates of aneuploidy for all chromosomes.

Results: In addition to confirming a maternal age effect, evidence suggested that embryos reaching a more advanced stage of development on day 5 were more likely to be chromosomally normal than those not reaching the same stage until day 6 . The poorer quality embryos were more likely to be aneuploid in the most age groups but this effect was seen more markedly in the trophectoderm (TE) than the inner cell mass.

Conclusions: When comparing aneuploidy to growth rate, a complex pattern emerges in that, in the whole data set, it is the slower growing embryos that appear to be more likely to be aneuploid (but only in the younger and older age groups). By isolating the slower growing cohort (those not ready to biopsy until day 6 ) however, it seems that the relatively faster ones that continue to grow to day 6 are more likely to be aneuploid. Moreover, TE quality appears to be an important consideration for choosing embryos in all age groups.
\end{abstract}

Keywords: Aneuploidy; Morphology; Kinetics; Embryo selection

\section{Introduction}

The ability to culture human embryos in-vitro, ultimately to lead to viable live births, has improved over the last 20 years, mostly through the evolution of culture media and methods. The development of physiological culture media similar to human tubal fluid improved culture conditions of human embryos [1] and further modifications using sequential media allowed for successful extended culture to the blastocyst stage [2,3]. Unlike the sophisticated development of culture media the method of choosing which embryos to transfer using basic morphological criteria has remained similar since the inception of IVF. That is, the subjective judgment of the embryologist is still the major driver in embryo selection and, if this aspect could be improved, there is still potential for further increases in IVF pregnancy rates. Other more empirical, approaches to inform embryo choice have, in recent years, gained in popularity: comprehensive chromosome screening (CCS) for aneuploidy, and morphokinetic analyses following timelapse photography.

There are a number of referral categories for CCS in a clinical IVF setting, advanced maternal age recurrent pregnancy loss, recurrent implantation failure and prolonged periods of infertility (e.g. related to elevated sperm aneuploidy). Of these the maternal age effect for aneuploidy has the most well-described in preimplantation embryos as well as oocytes, spontaneous abortions and live born individuals and thus remain the largest referral category for PGS [4,5]. Moreover, while the appearance of an embryo tells us which, among a cohort, may be the most likely to be viable in early embryonic stages, aspects of visual assessment have yet to be fully established as reasonably accurate predictors of ploidy status. Blastocyst biopsy followed by CCS for aneuploidy has been shown to reduce, but not eliminate the risk of miscarriage [6,7] when transferring chromosomally normal embryos and the chance of choosing a karyotypically normal embryo for transfer based on appearance alone becomes less likely as the patient

*Corresponding author: Griffin DK, School of Biosciences, University of Kent, Canterbury, Kent, CT2 7NJ, UK, Tel: 004401227 823022; E-mail: D.K.Griffin@kent.ac.uk

Received May 21, 2015; Accepted October 08, 2015; Published October 10, 2015

Citation: Coates A, Coate B, Holmes L, Griffin DK (2015) Morphological and Kinetic Embryological Criteria and Correlation with Aneuploidy Rates: How Migh they Be Used to Choose the Best IVF Embryo for Transfer? Human Genet Embryol 5: 129. doi:10.4172/2161-0436.1000129

Copyright: ( 2015 Coates A, et al. This is an open-access article distributed unde the terms of the Creative Commons Attribution License, which permits unrestricted use, distribution, and reproduction in any medium, provided the original author and source are credited. 
ages. Embryo biopsy followed by CCS thus remains the only method of accurately detecting aneuploidy in pre-implantation embryos. Studies involving transfer of chromosomally normal embryos have resulted in higher implantation rates per embryo transferred in older patients than transfer of non-tested embryos chosen by morphology alone [814]. A further aspect of CCS is that it tells us which chromosome is involved and this can be important in predicting obstetric outcome. For example, the absence of monsomies (apart from XO) among spontaneous abortion material suggests that monosomy is a leading cause of embryo arrest or implantation failure. The preponderance of trisomy of most chromosomes (and XO) in first trimester miscarriages makes aneuploidy the leading cause of pregnancy loss. The potential for stillbirth or congenital abnormalities in liveborns for certain aneuploidies (e.g. trisomy 21,22) make chromosome specific screening useful to minimize the likelihood of an aneuploid embryo being transferred to the uterus (reviewed in Griffin 1996).

Recent advances in time-lapse technology with cameras installed inside incubators have allowed constant, undisturbed observation of embryo growth patterns. Evidence suggests that timing of one cell division to the next at the early cleavage stage is somewhat predictive of blastocyst formation but not ploidy status [15-19]. Campbell and colleagues demonstrated that the most predictive morphokinetic events were the timing of the beginning to the end of blastulation and that was able to give an overall risk (i.e. low vs. high), of an embryo being aneuploid. Despite these impressive results, although we may be able to provide crude estimates of the risk of embryos being chromosomally normal or abnormal based on morphokinetics, further refinements are needed if non-invasive methods are to replace invasive ones for preimplantation genetic screening. In other words, there is still a need to investigate further morphological and morphokinetic parameters (either by static or time-lapse analysis) to establish which, if any, might be correlated with specific aneuploidy risk. While direct analyses of time-lapse images offers the potential to correlate these morphokinetic events and potentially establish thresholds and ranges within which a lab can predict ploidy status of an embryo, there is not a definitive event which predicts aneuploidy accurately.

While direct analyses of time-lapse images offer an opportunity to address the above questions, static analysis in the traditional manner nonetheless remains useful in identifying the criteria through which time-lapse studies might be directed. This is particularly true for clinics that have not yet installed a time-lapse facility and/or if preexisting data, that can be analyzed retrospectively, is used to identify factors that might pre-dispose to aneuploidy. Moreover, it is essential that such analysis is performed in specific maternal age windows, given the association of maternal age and aneuploidy. For this reason we have compared aneuploidy rates following CCS by static analysis of specific morphological and morphokinetic parameters; namely the quality of the tropectoderm and inner cell mass individually and the speed of growth as manifested by the day that the embryo achieves full expansion of the blastocoel cavity (i.e. is it "blastocyst biopsy ready" by day 5). Because of the link to maternal age, each analysis is performed on 5 distinct age groups.

\section{Materials and Methods}

Patients undergoing IVF treatment at Oregon Reproductive Medicine (ORM), Portland, Oregon, USA, were counseled to have their embryos tested as part of our routine protocol to assess the chromosomal status of pre-implantation embryos before transfer to the uterus. All embryos were created from super-ovulated IVF cycles to create multiple embryos. Chesapeake IRB review (September $6^{\text {th }}$ 2013) determined IRB approval was not required; the University of Kent Local Research Ethics Committee approved this study.

\section{Ovarian Stimulation}

Controlled Ovarian Hyperstimulation $(\mathrm{COH})$ protocols for IVF cycles included agonist and antagonist down-regulation protocols. One third of the patients underwent a GnRH agonist protocol using Lupron (leuprolide acetate: BDI Pharma) for down regulation and two thirds of the patients underwent GnRH antagonist (Ganerelix acetate, Merck) suppression. Both down regulation regimes were followed by ovarian stimulation for 9-12 days using a combination of injectable hMG (Menopur, Repronex, Ferring Pharmaceuticals) and injectable recombinant FSH (Gonal-F, Serono) to achieve maximum oocyte yield. All cycles received an hCG trigger of 5,000 or 10,000IU (Pregnyl/ Ovidrel or Novarel, Schering-Plough/Serono or Ferring) 35 hours prior to scheduled oocyte retrieval time, once at least 3 follicles reached $17 \mathrm{~mm}$ in diameter. Oocyte retrieval under ultrasound guidance was used to aspirate all mature follicles.

\section{Embryo Culture}

Oocytes were placed in Quinns Fertilization medium (Origio), supplemented with 5\% HSA (Irvine Scientific) and Intra-Cytoplasmic Injection (ICSI) or standard insemination was carried out 4 hours post retrieval. If standard. IVF was used, sperm still bound to the zona pellucida on day 5 had potential to contaminate the biopsy sample however once the trophectoderm has breached the zona, sperm are left behind bound to the zona and are easily avoided. Around 18-20 hours post retrieval oocytes were examined for the presence of pro-nuclei. Zygotes were placed into Quinns cleavage stage medium (Origio) supplemented with $10 \%$ HSA until day 3 of development. All cleaved embryos were transferred to Quinns Blastocyst medium supplemented with $10 \%$ HSA on day 3 of culture and remained there until transfer or vitrifcation on day 5 and or 6 of growth.

\section{Embryo Biopsy and Comprehensive Chromosome Screening}

All embryos to potentially be biopsied were hatched on day 3 post retrieval with a Hamilton Thorne laser making a small opening and left to day 5 of development. Embryos that grew to an expanded blastocyst stage had 3-6 TE cells excised using a Hamilton Thorne laser with an 800 um pulse, on day 5 or 6 of development. The biopsied cells were placed in wash buffer in microfuge tubes and labeled accordingly with appropriate embryo number. The biopsied embryos were then vitrified using Medicult DMSO free vitrification media (Medicult; Denmark) on cryotops (Kitazato inc, Tokyo, Japan) from November 2010 until December 2012 and then Irvine scientific vitrification media with DMSO post December 2012. Biopsied cells were sent to Reprogenetics laboratory LA, for analysis using array CGH (Bluegnome, Cambridge UK). Chromosome results were obtained around 2 days post biopsy. Chromosomally normal embryos were kept in storage for future use.

\section{Embryo Grading and Morphological Analysis}

Embryos were considered suitable for biopsy on day 5 ("day 5 ready") when at least $10 \%$ of the TE was protruding from the breach in the zona pellucida made on day 3 . All embryos not fully expanded by day 5 were cultured until day 6 and biopsied before noon if they reached full expansion by that time ("day 6 ready"). Embryos were only biopsied if there was a visible Inner cell mass (ICM) and multicelled TE protruding from the zona pellucida. Blastocysts were graded using the Gardner method [20]. This method relies on a 3 part grading 
system. A number indicates the degree of expansion of the blastocoel cavity and 2 following letters indicate the quality of the inner cell mass and the trophectoderm respectively. So a good quality blastocyst would be denoted as a 4AA, (fully expanded cavity=4; good quality $\mathrm{ICM}=\mathrm{A}$; good quality TE=A). Embryos were not biopsied if an ICM was not seen. "Early blastocyst" describes an embryo showing the first signs of blastulation and is not graded at this point. Compacting embryos with no visible sign of blastulation were classified as morulae, which is the stage before the embryo begins to cavitate and cell outlines begin to merge. Embryos classified as "poor quality" with a $\mathrm{C}$ grade of either ICM and/or TE were not typically biopsied and tested as we routinely only freeze embryos of average or good quality that have a good chance of implanting.

\section{Statistical Analysis}

Mann-Whitney (for 2 way comparison) and Kruskal-Wallis tests ( for 3 way comparison)(non-parametric ANOVA tests) were used to determine significance. $P$ values of $<0.05$ were considered statistically significantly different.

\section{Results}

In this study, a total of 1686 Embryos from 295 cycles of IVF were successfully biopsied; successful array CGH traces were seen in $97 \%$ of cases. Mean age, number of cycles and mean embryo biopsied per cycle are noted in Table 1. There was no significant difference in the proportion of embryos that did not give a result in each age group.

\section{Aneuploidy and Developmental Stage at a Fixed Time Point}

In the first analysis, for 4 of the age groups, we compared the overall aneuploidy rates of "day 5 ready" vs. "day 6 ready" embryos, the latter being the slower growing. Results in the donor egg (youngest) and $40+$

\begin{tabular}{|l|c|c|c|c|c|}
\hline Group number & $\mathbf{1}$ & $\mathbf{2}$ & $\mathbf{3}$ & $\mathbf{4}$ & $\mathbf{5}$ \\
\hline Age ID & $\begin{array}{c}\text { Donor } \\
\text { egg }\end{array}$ & $<35$ & $35-39$ & $40-42$ & $>42$ \\
\hline Mean age & 24.8 & 31.4 & 35.5 & 41 & 43.5 \\
\hline No. of cycles & 82 & 46 & 107 & 53 & 7 \\
\hline No. of embryos biopsied & 685 & 284 & 509 & 236 & 25 \\
\hline $\begin{array}{l}\text { Mean no. of embryos } \\
\text { biopsied/cycle }\end{array}$ & 8.4 & 6.2 & 4.8 & 4.5 & 3.6 \\
\hline \# embryos with no result & 15 & 12 & 16 & 8 & 1 \\
\hline
\end{tabular}

Table 1: All embryos used in this study broken down into maternal age groups.

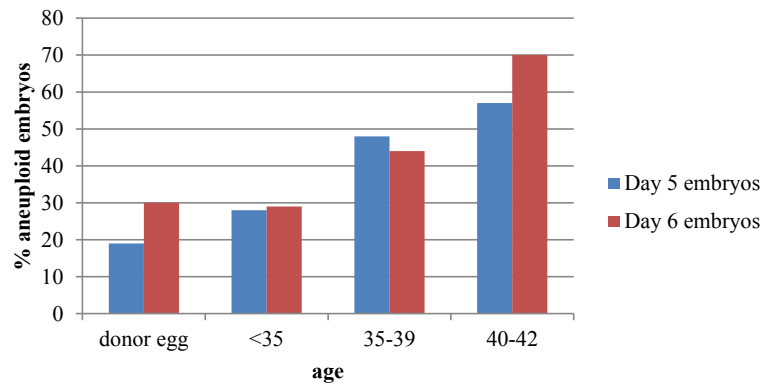

Figure 1: Aneuploidy rates vs. developmental stage. \%Aneuploidy rates (Y axis) of embryos according to the day they reached the stage of development ready to biopsy (day 5 or 6 ). Donor egg: $p=0.001$ (significantly different); $<35$ : $p=>0.05$ (not significant); 35-39: $p=>0.05$ (not significant); 40-42: $p=0.04$ (significantly different-Mann-Whitney test).

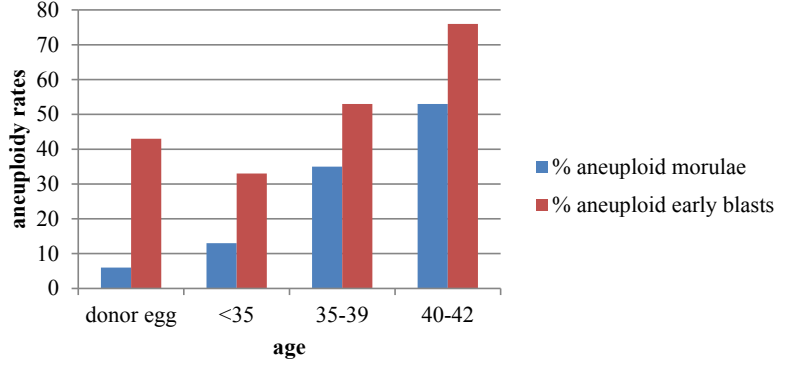

Figure 2: \%Aneuploidy rates (y axis) of embryos that were early blastocysts or morulae on day 5 . The aneuploidy rates of blastocysts resulting from morulae on day 5 appeared to be lower in each age group than aneuploidy rates from embryos that had just begun to cavitate on day 5 (early blastocysts). Donor egg: $p=0.009$ (significantly different); $<35: p=>0.05$ (not significant); 35-39: $p=0.08$ (not significant); 40-42: $p=0.08$ (not significant - Mann-Whitney)

oldest age groups show that faster growing embryos i.e. those that were structurally ready for biopsy on the morning of day 5 ("day 5 ready") were more likely to be chromosomally normal than ones that were slower growing and ready for biopsy by day 6 ("day 6 ready"). In the $<35$ and 35-39 groups rates of aneuploidy between the two groups were not signficantly different. There were too few "day 5 ready" embryos (only 2) in the $>42$ age group and therefore this group was not included for this particular analysis (Figure 1).

Results thus suggest an association between aneuploidy rate and speed of growth, but only in embryos of younger and older mothers.

Independent of age, "day 5 ready" and slower growing "day 6 ready" embryos showed a significant difference in frequency of aneuploidy, with faster growing embryos exhibiting an aneuploidy rate of $31 \%$ and slower growing embryos $41 \%$. This finding is very statistically significant $(\mathrm{p}=0.0001)$

In a second analysis a subset of the embryos not ready for biopsy until day 6 were taken forward for analysis (Figure 2). Aiming to correlate aneuploidy and speed of growth, levels of aneuploidy in each age group were compared. This particular subset of embryos was either morulae or early blastocysts on day 5 . They were individually tracked and the ones that subsequently grew to fully expanded blastocysts by day 6 were able to be biopsied. In all age groups the early blastocysts (quicker growing embryos) were more likely to be aneuploid than the morulae (slower growing). Again there were too few embryos biopsied (only 5) in the $>42$ age group and therefore this group was not included. This finding was statistically significant for the younger (donor egg) group only (although in the 35-39 and the 40-42 age groups the difference was borderline significant)

Taken together therefore, a complex pattern emerges in that, in the whole data set, it is the slower growing embryos that appear to be more likely to be aneuploid (but only in the younger and older age groups). By isolating the slower growing cohort (those not ready to biopsy until day 6) however, it seems that the relatively faster ones that continue to grow to day 6 are more likely to be aneuploid.

\section{Aneuploidy Rates Compared to Overall Embryo Grade}

For this analysis, the quality of the embryo was classified by the quality of its trophectoderm (TE) and inner cell mass (ICM) separately. With AA grade as the highest quality and $\mathrm{BB}$ the lowest for both germ layers $\mathrm{AB}$ or $\mathrm{BA}$ referred to embryos with one good quality layer. Embryos classified as good quality (AA) had the lowest rate of aneuploidy in all age groups: (19\%, 24\%, 42\%, 52\% and 57\% respectively) 


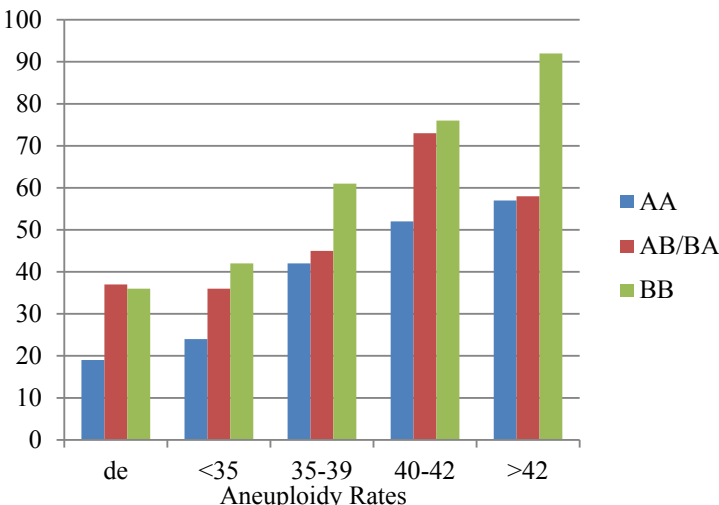

Figure 3: Rates of aneuploidy according to overall grade of embryos. Donor egg: $p=0.0001$ (significantly different), <35: $p=0.06$ (not significant); 35-39: $p=0.02$ (significantly different); 40-42: $p=0.006$ (significantly different); $>42$ $\mathrm{p}=0.04$ (significantly different - Kruskal-Wallis test).

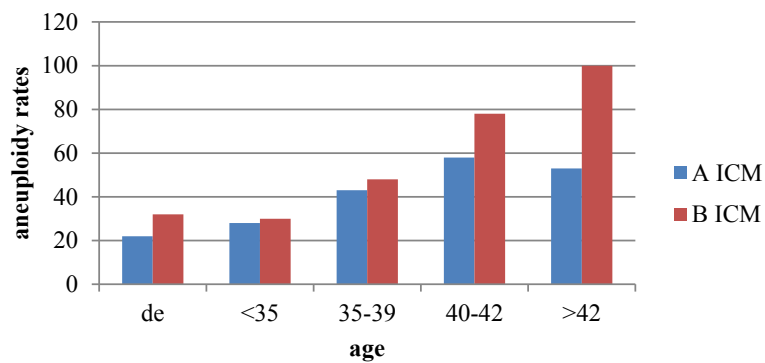

Figure 4: Inner cell mass (ICM) quality and rates of aneuploidy. Aneuploidy rates of embryos with a $\mathrm{B}$ grade ICM were significantly higher than A grade ICM in the donor egg group (average age 24 ) and the over 40 age groups. Donor egg: $p=0.048$ (significantly different); $<35: p=>0.05$ (not significant), 35-39: $p=>0.05$ (not significant), 40-42: $p=0.005$ (significantly different), $>42: p=0.03$ (significantly different - Mann-Whitney test).

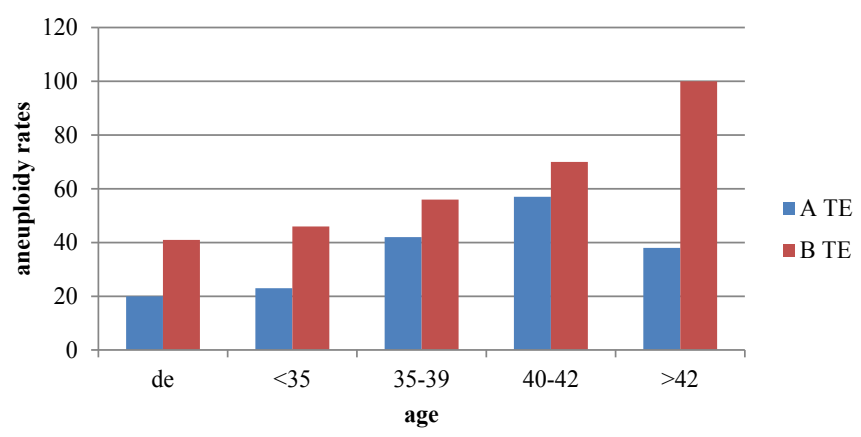

Figure 5: Rate of aneuploidy and quality of trophectoderm (TE). Aneuploidy rates of embryos with a $B$ grade TE were significantly higher than $A$ grade TE in all but one age groups. Donor egg: $p=0.0001 ;<35$ : $p=0.0007 ; 35-39$ : $p=0.039$; 40-42: $p=0.06$ (not significant); $>42: p=0.00$.

compared to embryos with at least one poorer quality part ( $\mathrm{AB}$ or $\mathrm{BA})$, which had higher rates $(37 \%, 36 \%, 45 \%, 73 \%$ and $58 \%$ respectively). Embryos with the poorest quality overall (BB) had the highest aneuploidy rates (36\%, 42\%, 61\%, 76\% and $92 \%$ respectively) (Figure 3).

\section{Inner Cell Mass and Trophectoderm Quality Compared to Aneuploidy}

The quality of the inner cell mass correlated with aneuploidy levels in three of the five age groups (the $<35$ and 35-39 age groups not being statistically significantly different). The quality of the trophectoderm correlated with aneuploidy status in four of the five age groups (the 4042 age group not quite reaching statistical significance $(\mathrm{p}=0.06))$. In all cases the aneuploidy levels in the B grade germ layers were higher than the A grade. Taken together therefore the results seem to suggest that TE quality is a more accurate predictor of aneuploidy (Figures 4 and 5).

\section{Discussion}

Choosing the embryo most likely to lead to a baby is the ultimate goal in any IVF cycle. The development of more physiological culture media $[1,2]$ has enabled successful growth of embryos to the blastocyst stage. Better culture conditions eliminate the cohort of embryos that arrest at day 3 and improves implantation rates per embryo. In our own hands (current data 2009-2012) implantation rates of fresh untested blastocyst embryos are typically $56 \%$ in patients $<35$ compared to $35 \%$ if day 3 embryos are transferred. However, although extended embryo culture narrows down the choice of embryos it still does not accurately predict which embryo to choose based on its appearance. Embryo grading by an experienced embryologist also remains a subjective art rather than an exact science. The difference between an A grade and a B grade TE or ICM can be open to interpretation and the exact grading of embryos is acknowledged to be of questionable utility.

A recent study illustrated how choosing an embryo based on appearance alone is a fairly arbitrary choice if there are many to choose from ref. [21]. Embryos were chosen using morphological criteria and then tested the embryos before transfer to see if the morphological choice was correct. The embryos were biopsied to assess chromosome complement and transferred on day 6 if the embryo was euploid. If the original choice was aneuploid another embryo would be selected for transfer. Selection of an aneuploid embryo by chance based on appearance alone was higher in patients $\geq 35$ compared to $<35$ years old ( $31 \%$ vs. $14 \%)$ purely because there is a higher percentage of chromosomally normal embryos in younger patients.

Morphology has been related to aneuploidy in a study of 500 embryos from 93 patients [11]. A correlation was found between embryo quality and ploidy status but this was not a strong correlation. That is, $50 \%$ of higher grade embryos were euploid compared to $37.5 \%$ of poorer quality embryos. Inner cell mass and trophectoderm grades were also affected by aneuploidy in a negative way. However some of the best quality embryos were aneuploid and some of the poorer quality embryos were chromosomally normal. A moderate relationship was shown in a retrospective study between blastocyst morphology and aneuploidy using CCS data [10] but implantation ability was governed by the chromosomal complement of an embryo rather than conventional morphology assessment. This is confirmed in data in this study.

Trophectoderm and ICM quality have been correlated with live birth outcome in two single embryo transfer studies. The conclusions were that TE quality was the most important factor in predicting live birth outcome but neither study specified the age of patients [22,23]. In the current study, TE quality appears to be more indicative of ploidy status than ICM quality whereas in patients 40 and older, ICM quality is more indicative of ploidy status when choosing embryos for transfer. However, when using a more detailed classification of ICM morphology, implantation potential has been predicted more accurately, with the shape and size being clinically important as a predictor of success [24]. Day 5 expanded blastocysts with slightly oval ICMs implanted at a higher rate (58\%) compared with those with either rounder ICMs (7\%) 
Citation: Coates A, Coate B, Holmes L, Griffin DK (2015) Morphological and Kinetic Embryological Criteria and Correlation with Aneuploidy Rates: How Might they Be Used to Choose the Best IVF Embryo for Transfer? Human Genet Embryol 5: 129. doi:10.4172/2161-0436.1000129

Page 5 of 6

or more elongated ICMs (33\%). Implantation rates were highest (71\%) for embryos with both optimal ICM size and shape. In the current study a detailed classification of the ICM was not performed and a gross overall appearance of the ICM as a distinct cohesive clump of cells was classed as a good quality ICM.

The technique for choosing embryos that perhaps has received the most attention in recent years is the study of morphokinetics. Precursors to the latest morphokinetic studies using time-lapse photography, involved taking the embryo out of the incubator at certain developmental junctions and observing if an event had occurred. One study showed that if the first cleavage division occurred before 25 hours post insemination an embryo was twice as likely to develop to an expanded blastocyst compared to an embryo that failed to cleave within 25 hours post insemination (32\% vs. 16\%) [17]. Recent advances in time-lapse technology have allowed constant, undisturbed observation of embryo growth patterns. Timing of early cleavage events predict with some accuracy embryos which will ultimately form a blastocyst but not embryos that are chromosomally normal [25-27]. The development of a risk classification system using time-lapse photography in conjunction with biopsy and chromosomal analysis showed that the timing from the start to the end of blastulation is predictive of ploidy status and resulted in live birth rates per embryo similar to those seen after replacing known euploid embryos (61\% with morphokinetics blastulation assessment vs. $69 \%$ using chromosome analysis) [28]. Interestingly using our own data, morulae on day 5 (pre-blastulation) that continued to grow to fully expanded blastocysts on day 6 had a much lower rate of aneuploidy than embryos that had just begun to blastulate on day 5. This supports previous findings [18] which found that delayed blastulation does not in itself result in higher rates of aneuploidy but that, if blastulation does not occur at all the rates of aneuploidy are high. Therefore if a morula carries on growing to an expanded blastocyst then it has a good chance of being chromosomally normal as shown by our own data. The convention has been to choose more advanced embryos using traditional static morphology for transfer from a cohort. If all that were available on day 5 were early blastocysts or morulae then an embryologist would be far more likely to choose the early blastocyst over a morula. One might conclude that if all that are available on day 5 are early blasts or morulae then maybe the transfer should be carried out on day 6 if the embryos continue to progress and choose for transfer the embryos that were at the morula stage on day 5 .

Our own data set suggests that, although there may well be correlations between growth rate and aneuploidy, the relationship is by no means a simple one. Finding, as we did, that faster growing embryos ready for biopsy by day 5 were more likely to be chromosomally normal than slower growing embryos not ready for biopsy until day 6 , suggests a simple correlation, albeit in only certain age groups. Finding an apparently paradoxical phenomenon when looking at the day 6 ready group only (i.e. that the ones that reached early blastocyst on day 5 were more likely to be chromosomally abnormal compared to embryos that had not yet begun to cavitate on day 5) however suggests a complex pattern that needs further investigation. In other words, growth rate criteria (whether determined by time lapse or, as in this case, by static criteria) need to be viewed with caution and skepticism when trying to draw conclusions about levels of aneuploidy.

In conclusion, it seems that there is yet to be found a morphokinetically based assessment that will predict with accuracy which embryo to choose without analyzing the chromosome complement of an embryo; this can currently only be achieved by embryo biopsy. Moreover, correlating the end point morphology of an embryo to its ploidy status tell us that embryos that we may have dismissed as poorer quality may often be euploid and embryos that we deem to be good quality may be aneuploid [10,11]. Static Embryo morphology assessment thus has a place in predicting embryo viability but has limitations in predicting the likelihood of an embryo being euploid. It is possible that we should be looking at different parameters for different patient populations, perhaps for instance choosing embryos with better quality ICMs for the $>40$ patients (if there is a choice) but focusing on the trophectoderm in other age groups. Time lapse evaluation of morphokinetic events should be viewed with caution when using that alone to attempt to decide ploidy status as further studies need to be performed to establish correlation between morphokinetic event timing and ploidy status [29]. A combination of chromosomal analysis and time-lapse observations of morphokinetic criteria or more detailed static morphological observations may help inform future studies aimed at choosing the best embryo for transfer. For the moment however, the use of morphology or growth rate for the prediction of aneuploidy remains an experimental approach.

\section{References}

1. Quinn P (1995) Enhanced results in mouse and human embryo culture using a modified human tubal fluid medium lacking glucose and phosphate. J Assist Reprod Genet 12: 97-105.

2. Marek D, Langley M, Gardner D, Confer N (1999) Introduction of blastocys culture and transfer for all patients in an in vitro fertilization program. Fertil Steril 72: $1035-1040$

3. Gardner DK, M Lane (1997) Culture and selection of viable blastocysts: a feasible proposition for human IVF? Hum Reprod Update 3: 367-382.

4. Harton G, Munne S, Surrey M, Grifo J, Kaplan B, et al. (2013) Diminished effect of maternal age on implantation after preimplantation genetic diagnosis with array comparative genomic hybridization. Fertil Steril 100: 1695-1703.

5. Qi S, LF Liang, YX Xian, JQ Liu, Wang W, et al. (2014) Arrested human embryos are more likely to have abnormal chromosomes than developing embryos from women of advanced maternal age. J Ovarian Res 7: 65.

6. Munne S, Chen S, Fischer J, Colls P, Zheng X, et al. (2005) Preimplantation genetic diagnosis reduces pregnancy loss in women aged 35 years and older with a history of recurrent miscarriages. Fertil Steril 84: 331-335.

7. Munne S, Fischer J, Warner A, Chen S, Zouves C, et al. (2006) Preimplantation genetic diagnosis significantly reduces pregnancy loss in infertile couples: a multicenter study. Fertil Steril 85: 326-332.

8. Scott RT, Upham KM, Forman EJ, Zhao T, Treff NR, et al. (2013) Cleavagestage biopsy significantly impairs human embryonic implantation potentia while blastocyst biopsy does not: a randomized and paired clinical trial. Fertil Steril 100: 624-630.

9. Hardarson T, Hanson C, Sjögren A, Lundin K (2001) Human embryos with unevenly sized blastomeres have lower pregnancy and implantation rates: indications for aneuploidy and multinucleation. Hum Reprod 16: 313-338.

10. Capalbo A, Rienzi L, Cimadomo D, Maggiulli R (2014) Correlation between standard blastocyst morphology, euploidy and implantation: an observational study in two centers involving 956 screened blastocysts. Hum Reprod 29: 1173-1181.

11. Alfarawati S, Fragouli E, Colls P, Stevens J (2011) The relationship between blastocyst morphology, chromosomal abnormality, and embryo gender. Fertil Steril 95: 520-524.

12. Schoolcraft W, Treff NR, Stevens JM, Ferry K (2011) Live birth outcome with trophectoderm biopsy, blastocyst vitrification, and single-nucleotide polymorphism microarray-based comprehensive chromosome screening in infertile patients. Fertil Steril 96: 638-640.

13. Scott RT,Ferry K,Su J,Tao X, Scott K, et al. (2012) Comprehensive chromosome screening is highly predictive of the reproductive potential of human embryos: a prospective, blinded, nonselection study. Fertil Steril 97: 870-875.

14. Yang Z, Liu J, Collins GS, Salem SA, Liu X (2012) Selection of single blastocysts for fresh transfer via standard morphology assessment alone and with array CGH for good prognosis IVF patients: results from a randomized pilot study. Mol Cytogenet 5: 24 
Citation: Coates A, Coate B, Holmes L, Griffin DK (2015) Morphological and Kinetic Embryological Criteria and Correlation with Aneuploidy Rates: How Might they Be Used to Choose the Best IVF Embryo for Transfer? Human Genet Embryol 5: 129. doi:10.4172/2161-0436.1000129

Page 6 of 6

15. Chavez S, Loewke KE, Han J, Moussavi F (2012) Dynamic blastomere behaviour reflects human embryo ploidy by the four-cell stage. Nat Commun 3: 1251.

16. Ebner T, Moser M, Sommergruber M, Tews G (2003) Selection based on morphological assessment of oocytes and embryos at different stages of preimplantation development: a review. Hum Reprod Update 9: 251-262.

17. Fenwick J, Platteau P, Murdoch AP, Herbert M (2002) Time from insemination to first cleavage predicts developmental competence of human preimplantation embryos in vitro. Hum Reprod 17: 407-412.

18. Kroener L, Ambartsumyan G, Briton-Jones C (2012) The effect of timing of embryonic progression on chromosomal abnormality. Fertil Steril 98: 876-880.

19. Lundin K, Bergh C, Hardarson T (2001) Early embryo cleavage is a strong indicator of embryo quality in human IVF. Hum Reprod 16: 2652-2657.

20. Gardner DK, Schoolcratf W (1999) In vitro culture of human blastocysts, in Toward Reproductive Certainty: Fertility and Genetics Beyond. Parthenon Publishing London, UK 378-388.

21. E, Upham K, Cheng M, Zhao T, Hong K (2013) Comprehensive chromosome screening alters traditional morphology-based embryo selection: a prospective study of 100 consecutive cycles of planned fresh euploid blastocyst transfer. Fertil Steril 100: 718-724.

22. Ahlstrom A, Westin C, Reismer E, Wikland M (2011) Trophectoderm morphology: an important parameter for predicting live birth after single blastocyst transfer.
Hum Reprod 26: 3289-3296.

23. Hill M, Richter K, Heitmann R, Graham J (2013) Trophectoderm grade predicts outcomes of single-blastocyst transfers. Fertility and sterility 99: 1283-1289.

24. Richter K, Harris D, Daneshmand S, Shapiro B (2001) Quantitative grading of a human blastocyst: optimal inner cell mass size and shape. Fertil Steril 76 1157-1167.

25. Herrero J, Meseguer M (2013) Selection of high potential embryos using timelapse imaging: the era of morphokinetics. Fertility and sterility 99: 1030-1034.

26. Meseguer M, Rubio I, Cruz M, Basile N, Marcos J, et al. (2012) Embryo incubation and selection in a time-lapse monitoring system improves pregnancy outcome compared with a standard incubator: a retrospective cohort study. Fertil Steril 98: 1481-1489 e10.

27. Paternot G, Debrock S, Neubourg D (2013) Semi-automated morphometric analysis of human embryos can reveal correlations between total embryo volume and clinical pregnancy. Hum Reprod 28: 627-632.

28. Campbell A, Fishel S, Laegdsmand M (2014) Aneuploidy is a key causa factor of delays in blastulation: author response to 'A cautionary note against aneuploidy risk assessment using time-lapse imaging'. Reprod Biomed Online 28:279-283.

29. Ottolini C, Rienzi L, Capalbo A (2014) A cautionary note against embryo aneuploidy risk assessment using time-lapse imaging. Reprod Biomed Online 28: $273-275$ 